\title{
Thickness variations in extraordinary and spontaneous Hall coefficients of iron films
}

\author{
C. R. Pichard, C. R. Tellier, A. J. Tosser and C. Vautier $\left(^{*}\right)$ \\ Laboratoire d'Electronique, Université de Nancy - I, C.O. 140, 54037 Nancy-Cedex, France
}

(Reçu le 28 janvier 1980, révisé le 31 mars 1980, accepté le 16 avril 1980)

\begin{abstract}
Résumé. - On présente les résultats de l'étude des variations des coefficients de Hall extraordinaire $\left(R_{1}\right)$ et spontané $\left(R_{\mathrm{s}}\right)$ avec l'épaisseur $d$ de couches de fer d'épaisseurs comprises entre 100 et $1000 \AA ̊$. Il apparaît que les courbes traduisant les variations dimensionnelles des produits $R_{1} . a$ et $R_{\mathrm{s}} . a$ sont des droites, ce qui peut s'interpréter à partir des variations dimensionnelles de la résistivité totale $\rho$ des couches dues aux collisions internes (phonons) et aux réflexions simultanées des électrons de conduction sur les interfaces des couches, les joints de grains et autres types d'imperfections et impuretés. Une telle interprétation est conforme aux prédictions de certaines études théoriques relatives aux métaux massifs ferromagnétiques $\left(R_{\mathrm{s}} \sim \rho^{2}\right)$.
\end{abstract}

\begin{abstract}
The thickness, $d$, dependence of the extraordinary $\left(R_{1}\right)$ and spontaneous $\left(R_{\mathrm{s}}\right)$ Hall coefficients of iron films was studied in the range 100-1 000 $\AA$. The $R_{1} . a$ and $R_{\mathrm{s}} . a$ versus $a$ curves exhibit a straight line behaviour; this is interpreted in terms of size effects in the total film resistivity in agreement with some theoretical studies on the Hall effect in bulk ferromagnetics which have predicted that the SHC is proportional to the square of the total resistivity.
\end{abstract}

1. Introduction. - In the last 15 years a voluminous literature on ferromagnetic films has appeared; the largest portion of the experimental results has been devoted to the study of the magnetic anisotropies present in the film [1-9], the local deviations of the magnetization $\mathbf{M}[1,2,3,10,11]$ and the domain configuration of films $[1,2,3,12,13,14]$ whereas the theoretical developments of the magnetic anisotropy, the magnetization ripple and the possible domain structure for thin ferromagnetic films have been reviewed by several authors $[1,2,3,10]$.

Some workers $[15,16]$ have suggested that the Hall effect constitutes a convenient way to study magnetic properties since it is well established that the Hall field per unit current density in ferromagnetics varies with the state of magnetization of the sample $[17,18]$. Some studies [16, 19-29] have therefore been carried out to determine both the ordinary Hall coefficient (OHC) $R_{\mathrm{H}}$, the extraordinary Hall coefficient (EHC) $R_{1}$ and the saturation magnetization $M_{\mathrm{s}}$. Most of these published data $[20,21,22,24,25,28,29]$ study the change in EHC with temperature $T$; the thickness

$\left.{ }^{*}\right)$ L.E.C.A.P., Université de Haute-Normandie, 76130 MontSaint-Aignan, France. dependence of the EHC has also been studied by Vautier [22], Le Bas [24], Coren et al. [16]; recently Wedler et al. [27] reported an unexpected oscillatory behaviour of the OHC and EHC. Coren and Juretschke performed measurements at room temperature on very thin films ( 20 to $100 \AA$ thick); since the films are generally discontinuous [30 to 34] at such thicknesses, it is surprising that these authors used the wellknown Fuchs-Sondheimer theory (F. S. theory) [35] which applies only to continuous metallic films; some additional details on the film structure are then needed to discuss these results.

It thus appears that the correlation between the thickness dependence of resistivity and EHC (or SHC, spontaneous Hall coefficient) has never been established for continuous films, a tentative interpretation is made in the present paper for continuous iron films whose thickness varies from 100 to $1000 \AA$.

2. Theoretical considerations. -2.1 THE FILM RESISTIVITY. - For thin metal films in which size effect arises because of the geometrical limitation of the mean free path by the film surface, the film resistivity $\rho_{\mathrm{F}}$ in absence of applied magnetic field may be written in the general form [35]

$$
\rho_{\mathrm{F}}=\rho_{\infty}+\rho_{\mathrm{s}}=\rho_{\infty}+C d^{-1}
$$


where $d$ is the film thickness, $\rho_{\infty}$ the resistivity of an infinitely thick film; and $\rho_{\mathrm{s}}=C d^{-1}$ the resistivity due to surface scattering; the constant $C$ depends upon the film material :

$$
C=\rho_{\infty} l_{0} \frac{3(1-p)}{8}
$$

where $l_{0}$ is the background mean free path and $p$ the specularity parameter [35].

For thin films in which the grain-boundaries act as efficient scatters, some recent theoretical studies $[36,37,38]$ have shown that the film resistivity may be written as

$$
\rho_{\mathrm{F}}=\rho_{\mathrm{g}}+\rho_{\mathrm{s}}
$$

where $\rho_{\mathrm{g}}$, the resistivity inclusive to the grain-boundary, is given by the Mayadas-Shatzkes function [36] and $\rho_{\mathrm{s}}$ is calculated dating from equation (2), [35].

However in addition to film surfaces and grainboundaries, impurities and frozen-in structural defects could also scatter the carriers; then according to the Matthiessen's rule we write in the more general case

$$
\rho_{\mathrm{F}}=\rho_{\mathrm{i}}+\rho_{\mathrm{g}}+\rho_{\mathrm{s}}
$$

where $\rho_{\mathrm{i}}$ is the contribution to resistivity of imperfections, including impurities but excluding grainboundaries; the form of equation (4) agrees well with that of equation (1) if the resistivity $\rho_{\mathrm{i}}$ does not show thickness dependence.

It should be also noted that some studies [30, 40] have shown that, in presence of small transverse magnetic field, the metal film resistivity can be approximately expressed by the theoretical expression (Eq. (1) or (3)) obtained from the F. S. or grain-boundary scattering conduction models.

2.2 The spontaneous Hall effect. - Among the set of various notations and definitions used in theoretical works $[17,18,41-45]$ we have chosen for convenience the notations of Hurd [18]. Then the Hall resistivity $\rho_{\mathbf{H}}$ is expressed in terms of applied magnetic induction $B$ and magnetization $M$ as

$$
\rho_{\mathrm{H}}=R_{\mathrm{H}} B+R_{\mathrm{s}} M
$$

and the SHC $R_{\mathrm{s}}$ is related to the EHC $R_{1}$ by equation

$$
R_{\mathrm{s}}=R_{1}-R_{\mathrm{H}} .
$$

We may note that, according to the Wannier model [46], some authors have introduced [17, 41] the Hall coefficient $R_{1}^{\prime}$

$$
R_{1}^{\prime}=R_{1}-\frac{1+P}{2} R_{\mathrm{H}} .
$$

However the quantity $P$ which measures the relative probability of a conduction electron to fall with magnetic momentum carrier, is of the order of the unity, then the difference between $R_{1}^{\prime}$ and $R_{\mathrm{s}}$ is very small at ordinary temperatures.

In the past years several attempts [41-45] have been made to explain the extraordinary Hall effect in ferromagnetic metals. In the scope of the present paper we are simply concerned with the results of these models [41-45] on the relationship between $R_{\mathrm{s}}$ and the electrical resistivity. In the Karplus and Luttinger model [41] it appears that $R_{\mathrm{s}}$ should vary with the square of the total electrical resistivity, i.e.

$$
R_{\mathrm{s}} \simeq A \rho^{2}
$$

where $A$ is a constant depending upon the metal and being theoretically thickness independent.

Equation (8) also agrees with theoretical analysis of Smit [42], Luttinger [43], Irkin and Shavrov [44] and Berger [45].

Inserting equation (1) into equation (8) gives

$$
R_{\mathrm{s}} \simeq A\left(\rho_{\infty}+C d^{-1}\right)^{2} .
$$

Since it is generally observed for relatively thick metallic films [47] that the quantity $C d^{-1}$ takes values which are negligible with respect to that of $\rho_{\infty}$, equation (9) becomes

$$
R_{\mathrm{s}} \simeq A\left(\rho_{\infty}^{2}+2 \rho_{\infty} C d^{-1}\right) .
$$

Noting that the SHC of an infinitely thick film is

$$
R_{\mathrm{s} \infty} \simeq A \rho_{\infty}^{2}
$$

equation (10) can then be rewritten in the form

$$
R_{\mathrm{s}} d=R_{\mathrm{s} \infty} d+2 R_{\mathrm{s} \infty} \rho_{\infty}^{-1} C .
$$

Equation (1) shows that a plot of $\rho_{\mathrm{F}} d$ versus $d$ should yield a straight line with the intercept as $C$ and the slope as $\rho_{\infty}$; similarly, from equation (12) a plot of $R_{\mathrm{s}} d$ versus $d$ should give a straight line with the ordinate intercept $2 R_{\mathrm{s} \infty} \rho_{\infty}^{-1} C$ and a slope $R_{\mathrm{s} \infty}$.

From the values of $\rho_{\infty}, C$ and $R_{\mathrm{s} \infty}$, determined from. the curves $\rho_{\mathrm{F}} d=f(d)$ and $R_{\mathrm{s}} d=f(d)$ the intercept of the $(R d, d)$ plot can be predicted.

3. Experimentals. - 3.1 EXPERIMENTAL RESUlTS. —Iron films, from 100 to $1000 \AA$ thick, were deposited by vacuum deposition and annealed at $480{ }^{\circ} \mathrm{C}$. Resistivity and Hall coefficient measurements were performed in situ [22]; details on the method of deposition and measurements have been given elsewhere [22].

Plot of $\rho_{\mathrm{F}} d$ versus $d$ illustrates the thickness dependence of the room temperature resistivity of thin iron films (Fig. 1).

The slope is

$$
\rho_{\infty} \approx 3 \times 10^{-7} \Omega . \mathrm{m}
$$




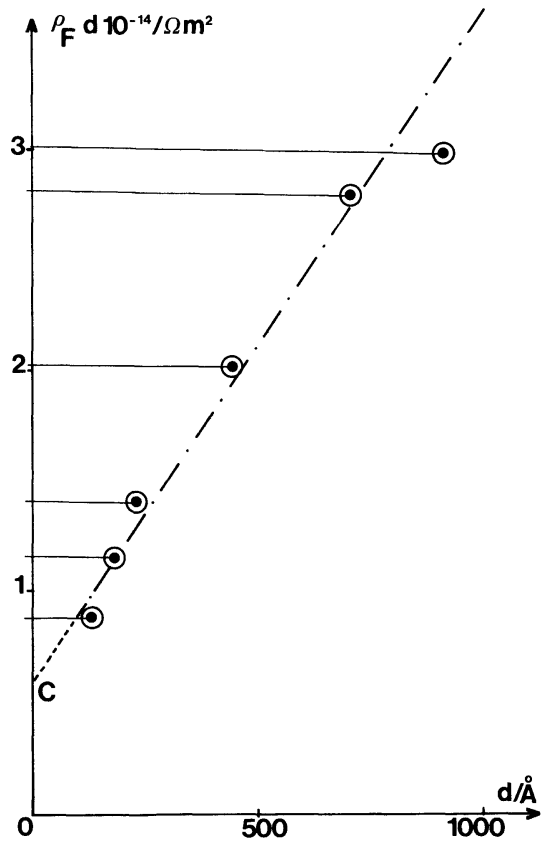

Fig. 1. - The product of the room temperature resistivity $\rho_{\mathrm{F}}$ by the thickness $d$ as a function of thickness $d$.

and the ordinate intercept is

$$
C \approx 0.6 \times 10^{-14} \Omega \cdot \mathrm{m}^{2} .
$$

Plots $R_{\mathrm{sF}} d$ and $R_{1 \mathrm{~F}} d$ versus $d$ are shown on figure 2 . As expected from equation (12) a straight line plot is obtained for $R_{\mathrm{s}} d$; the slope yield

$$
R_{\mathrm{s} \infty}=1.1 \times 10^{-9} \mathrm{~m}^{3} / \mathrm{A} . \mathrm{s}
$$

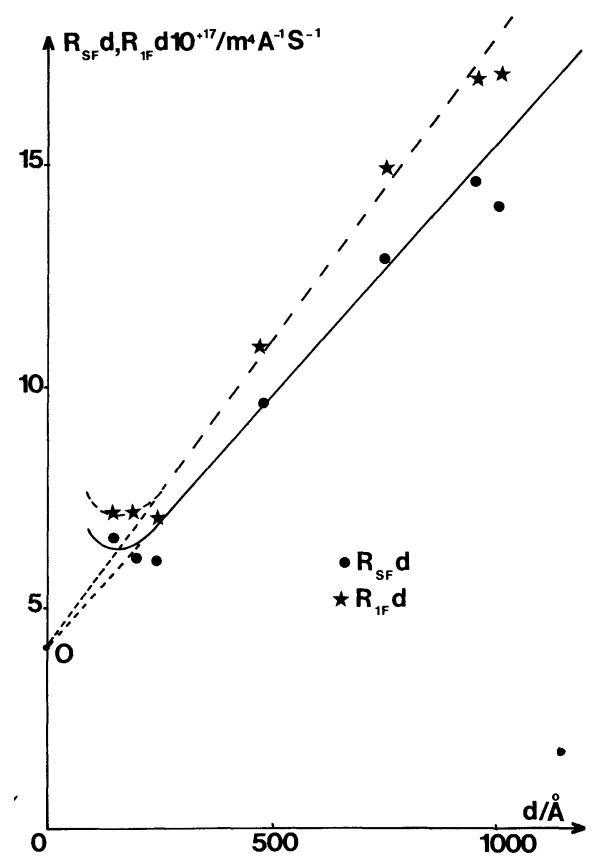

Fig. 2. - The product of the extraordinary Hall coefficient $R_{1 \mathrm{~F}}$ and spontaneous Hall coefficient $R_{\mathrm{sF}}$ by the thickness $d$ as a function of thickness $d$. and the experimental value of the ordinate intercept is equal to $4.2 \times 10^{-17} \mathrm{~m}^{4} /$ A.s.

Using the experimental values of $\rho_{\infty}, C$ and $R_{\mathrm{s} \infty}$ determined above, the calculated value of intercept is found to be

$$
0.44 \times 10^{-16} \mathrm{~m}^{4} / \mathrm{A} . \mathrm{s}
$$

this value is closed to the experimental value within the experimental errors.

It is also seen (Fig. 2) that the $\left(R_{\mathrm{s}} d, d\right)$ and $\left(R_{1} d, d\right)$ plots exhibit the same ordinate intercept; this feature may be easily interpreted by the fact that the thickness dependence of the OHC is only marked at small thickness according to classic size effect theories $[35,48]$; moreover, a nearly constant value

$$
R_{\mathrm{H}}=2.9 \times 10^{-10} \mathrm{~m}^{3} / \mathrm{A} . \mathrm{s}
$$

has been obtained [22] for iron films of thickness $d>450 \AA$.

The slopes $R_{1 \infty}$ and $R_{\mathrm{s} \infty}$ of the plots of figure 2 yield a value for the OHC $R_{\mathrm{H} \infty}$ of an infinitely thick film

$$
R_{\mathrm{H} \infty}=R_{1 \infty}-R_{\mathrm{s} \infty}=2.6 \times 10^{-10} \mathrm{~m}^{3} / \mathrm{A} . \mathrm{s} .
$$

This experimental value does not markedly deviate from the value previously measured on thick iron films [22].

The observed $R_{\mathrm{sF}} d$ versus $d$ plot shows some departure from the straight line relation at small thicknesses. This is attributed to the fact that the approximation $\left(\rho_{\infty} \gg C d^{-1}\right)$ is not satisfied in this thickness range.

3.2 Discussion. - From the experimental value of $C$ we may evaluate the experimental value of the background mean free path $l_{0}$ according to $\mathrm{F}$. S. or grain-boundary theories $[35,36,49]$; the obtained value is much too large and it must be assumed that $\rho_{\mathrm{i}}$ exhibits thickness variations; nevertheless if the total thickness dependent effect can be described by a linear relation (Fig. 1) the conclusions concerning the

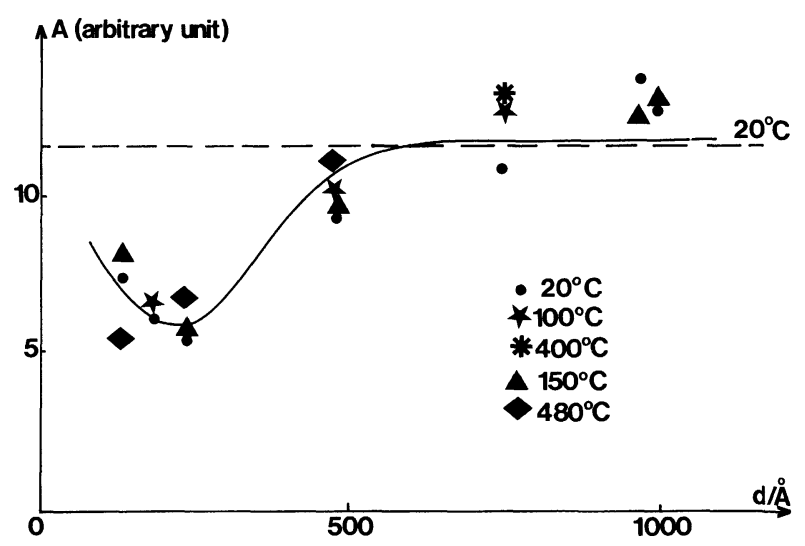

Fig. 3. - The thickness dependence of $A$. 
adequate of equation (12) for the description of the SHC size effects are not altered : the thickness dependence of the SHC can be represented with a satisfactory agreement by equation (9) or its approximate form equation (12) and $R_{\mathrm{s}}$ is proportional to the square of the total film resistivity due to both impurities, imperfections, grain-boundaries and surface scattering processes.

The coefficient $A$ (Eq. (8)) is generally assumed to be temperature independent [41] but it appears now that it must be the same for film of various thickness.
Evaluating $A$ from the measurements performed at different temperatures [22], it can be seen that these conditions are verified for $d>450 \AA$ (Fig. 3); this thickness range corresponds, as mentioned in section 3.1, to the best fit for the thickness dependence of $R_{\mathrm{s}}$.

4. Conclusion. - The SHC of thin annealed iron films shows a thickness dependence which may be described with good agreement by a $\rho^{2}$ law, where $\rho$ is the total film resistivity.

\section{References}

[1] Cohen, M. S., in Thin Film Phenomena by K. L. Chopra (Mc Graw-Hill, New-York) Chap. X, 1969.

[2] Prutton, M., Thin Ferromagnetic Films (Butterworths) London, 1964.

[3] Middleton, B. K., in Active and Passive Thin Film Devices (Academic Press, London) Chap. 11, 1978.

[4] Srivastava, R. S., J. Appl. Phys. 48 (1977) 1355.

[5] АвоAf, J. A., Kobliska, A. J. and Klokholm, E., IEEE Trans. Magn. 14 (1978) 941.

[6] Kobliska, R. J., Aboaf, J. A., Gangulee, A., Cuomo, J. J. and Klokholm, E., Appl. Phys. Lett. 33 (1978) 473.

[7] Chapman, V. B., Marwaha, A. S. and Collins, A. J., Thin Solid Films 58 (1979) 247.

[8] Bursuc, I. D., Calugaru, G., Thin Solid Films 5 (1970) 105 .

[9] Fisher, J. E., Thin Solid Films 5 (1970) 53.

[10] Leaver, K. D., Thin Solid Films 2 (1968) 149.

[11] Hoffmann, H., Thin Solid Films 58 (1979) 223.

[12] Blades, J. D. and Drew, J. B., Thin Solid Films 1 (1968) 135.

[13] Palatnik, L. S., Lubyanyi, L. Z. and Lukashenko, L. I., Sov. Phys. Solid State 15 (1973) 419.

[14] Tutovan, V., Velican, N., Thin Solid Films 7 (1971) 219.

[15] Foner, S., Phys. Rev. 95 (1954) 652.

[16] Coren, R. L. and Juretschke, H. J., J. Appl. Phys. 28 (1957) 806.

[17] JAN, J. P., in Solid State Physics, Ed. F. Seitz and D. Turnbull (Academic Press, New York) Vol. 5, (1959), p. 74.

[18] Hurd, C. M., The Hall Effect in Metals and Alloys (Plenum Press) Chap. 5 (1972).

[19] Vautier, C., Bordes, G. and Colombani, A., C. R. Hebd. Séan. Acad. Sci. 255 (1962) 906.

[20] Colombani, A., Vautier, C., C. R. Hebd. Séan. Acad. Sci. 262 (1966) 67.

[21] Vautier, C. and Colombani, A., C. R. Hebd. Séan. Acad. Sci. 262 (1966) 138.

[22] Vautier, C., J. Physique 27 (1966) 531.

[23] Le Bas, J., Thin Solid Films 10 (1972) 429.
[24] Le Bas, J., Publications de l'Université de Rouen (1967) p. 9. [25] Raeburn, S. J. and Aldridge, R. V., J. Phys. F. 8 (1978) 1917.

[26] Wedler, G. and Schneck, H., Thin Solid Films 47 (1977) 137.

[27] Wedler, G. and Schneck, H., Thin Solid Films 47 (1977) 147.

[28] Van Gurp, G. J., J. Phys. Chem. Phys. 38 (1977) 627.

[29] Aldridge, R. V., Thin Solid Films 60 (1979) 321.

[30] Chopra, K. L., Bobb, L. C. and Francombe, M. H., J. Appl. Phys. 34 (1963) 1699.

[31] Singh, B., Surplice, N. A., Thin Solid Films 10 (1972) 243.

[32] Adamov, M., Perovic, B. and Nenadovic, T., Thin Solid Films 24 (1974) 89.

[33] Michon, P., Thin Solid Films 16 (1973) 335.

[34] Suri, R., Thakoor, A. P. and Chopra, K. L., J. Appl. Phys. 46 (1975) 2574.

[35] Sondheimer, E. H., Adv. Phys. 1 (1952) 1

[36] Mayadas, A. F. and Shtazkes, M., Phys. Rev. B 1 (1970) 1382.

[37] Tellier, C. R., Thin Solid Films 51 (1978) 311.

[38] Pichard, C. R., Tellier, C. R. and Tosser, A. J., J. Phys. D 12 (1979) 101.

[39] Tellier, C. R. and Tosser, A. J., Revue Phys. Appl. 13 (1978) 441.

[40] Tellier, C. R., University of Nancy-I, Note interne (1979).

[41] Karplus, R. and Luttinger, J. M., Phys. Rev. 95 (1954) 1154.

[42] Smit, J., Physica 24 (1958) 739.

[43] Luttinger, J. M., Phys. Rev. 112 (1958) 739.

[44] Irkhin, Yu. P. and Shavrov, V. G., Sov. Phys. JeTP 15 (1962) 854.

[45] Berger, L., Phys. Rev. B 2 (1970) 4559.

[46] Wannier, G., Phys. Rev. 72 (1947) 304.

[47] See for example ChOPRA, K. L., Thin Film Phenomena (McGrau Hill, New York) Chap. 10 (1969).

[48] Tellier, C. R., Rabel, M. and Tosser, A. J., J. Phys. F 8 (1978) 2357.

[49] Tellier, C. R., Pichard, C. R. and Tosser, A. J., Le Vide' $196 \mathrm{~S}$ (1979) 93. 